\title{
Caracterização da Qualidade de Água e Sedimento na UHe americana Relacionados à Ocorrência de Plantas Aquáticas ${ }^{1}$
}

\author{
Characterization of Water and Sediment Quality at the Americana Reservoir Related to the \\ Occurrence of Aquatic Plants
}

\author{
VELINI, E.D. ${ }^{2}$, NEGRISOLI, E. ${ }^{3}$, CAVENAGHI, A.L. ${ }^{4}$, CORREA, M.R. ${ }^{3}$, BRAVIN, L.F.N. ${ }^{5}$, \\ DE MARCHI, S.R. ${ }^{3}$, TRINDADE, M.L.B. ${ }^{6}$, ARRUDA, D.P. ${ }^{7}$ e PADILHA, F.S. ${ }^{8}$
}

\begin{abstract}
RESUMO - Este trabalho foi realizado na UHE Americana, pertencente à Companhia Paulista de Força e Luz, e faz parte de um projeto de pesquisa e desenvolvimento realizado em conjunto com a Faculdade de Ciências Agronômicas (UNESP) de Botucatu. Foram realizadas amostragens de água e sedimento nos meses de outubro e dezembro de 2003 e fevereiro, abril e junho de 2004. Selecionaram-se seis pontos de coleta no reservatório, sendo cinco a montante da barragem e um a jusante. Levantamentos de flora foram realizados nos meses de dezembro de 2003 e abril e julho de 2004, sendo constatados elevados teores de nitrogênio e fósforo nas amostras de água, com valores médios de 3,867 $\mathrm{mg} \mathrm{L}^{-1}$ para nitrato, 0,706 $\mathrm{mg} \mathrm{L}^{-1}$ para amônia, 1,372 $\mathrm{mg} \mathrm{L}^{-1}$ para nitrito e $151,979 \mu \mathrm{g} \mathrm{L}^{-1}$ para fosfato dissolvido na água. O sedimento apresentou elevado nível de fertilidade, com médias de 32,18 $\mathrm{g} \mathrm{kg}^{-1}$ para matéria orgânica, 68,87 $\mathrm{mg} \mathrm{dm}^{-3}$ para $\mathrm{P}, 36,96 \mathrm{mmol} \mathrm{dm}^{-3}$ para Ca e $11,88 \mathrm{mmol} \mathrm{dm}^{-3}$ para $\mathrm{Mg}$. Espécies marginais e flutuantes foram as principais infestantes do reservatório, destacando-se Brachiaria subquadripara, Eichhornia crassipes, Pistia stratiotes e Salvinia auriculata.
\end{abstract}

Palavras-chave: plantas aquáticas, qualidade de água, sedimentos, reservatório.

\begin{abstract}
This work was carried out at the Americana Reservoir, owned by Companhia Paulista de Força e Luz, and was part of a joint $R \& D$ project with Faculdade de Ciências Agronômicas Botucatu - São Paulo - Brazil. Water and sediment samplings were collected in October and December 2003 and February, April and June 2004. Six sampling points were selected, being five upstream the barrage and one downstream. Flora assessment was carried out in December 2003, April and July 2004. High levels of nitrogen and phosphorus were observed in the water samples with average values of $3.867 \mathrm{mg} \mathrm{L}^{-1}$ for nitrate, $0.706 \mathrm{mg} \mathrm{L}^{-1}$ for ammonia, $1.372 \mathrm{mg} \mathrm{L}^{-1}$ for nitrite and $151.979 \mu \mathrm{g} \mathrm{L}^{-1}$ for phosphate dissolved in water. Sediment presented a high level of fertility with mean values of $31.18 \mathrm{~g} \mathrm{~kg}^{-1}$ for organic matter, $68.87 \mathrm{mg} \mathrm{dm}^{-3}$ for phosphorus, $36.96 \mathrm{mmol} \mathrm{dm}^{-3}$ for calcium and $11.86 \mathrm{mmol} \mathrm{dm}^{-3}$ for magnesium. Marginal and floating plants were the main weeds in the reservoir, with the most prominent being Brachiaria subquadripara, Eichhornia crassipes, Pistia stratiotes and Salvinia auriculata.
\end{abstract}

Key words: aquatic plants, water quality, sediments, reservoir.

1 Recebido para publicação em 25.1.2005 e na forma revisada em 24.3.2005.

2 Professor Doutor, Departamento de Produção Vegetal, FCA/UNESP, 18603-970 Botucatu-SP, <velini@uol.com.br>; ${ }^{3}$ PósGraduação, Departamento de Produção Vegetal, FCA/UNESP, Botucatu-SP; ${ }^{4}$ Eng.-Agr. Dr., Departamento de Produção Vegetal, FCA/UNESP, Botucatu-SP. ${ }^{5}$ Professor M.S. da Faculdade de Tecnologia de Botucatu, Botucatu-SP. ${ }^{6}$ Criativa, Consultoria em Matologia e Meio Ambiente. ${ }^{7}$ Químico, Departamento de Produção Vegetal, FCA/UNESP, Botucatu-SP. ${ }^{8}$ Companhia Paulista de Força e Luz. 


\section{INTRODUÇÃO}

Nos últimos anos tem se observado uma redução na quantidade e qualidade da água nos rios e reservatórios, com efeito direto na capacidade de geração de energia elétrica e abastecimento humano. As atividades desenvolvidas nas bacias hidrográficas que formam os reservatórios influenciam diretamente a qualidade da água e o tempo útil destes. A erosão da camada fértil de solos agrícolas e a grande descarga de efluentes residenciais e industriais têm levado rios e reservatórios, naturais ou artificiais, a uma condição de desequilíbrio, caracterizado pela grande disponibilidade de nutrientes na coluna d'água e no sedimento. Além de interferir decisivamente na qualidade da água, a grande disponibilidade de nutrientes pode levar ao crescimento desordenado de populações de plantas aquáticas.

As plantas aquáticas desempenham importantes funções na manutenção do equilíbrio natural de ambientes aquáticos (Tanaka, 1998; Thomaz, 2002), porém os inúmeros beneficios começam a se transformar em problemas quando elas deixam de coexistir em equilíbrio nesses ambientes (Tanaka, 1998; Velini, 1998; Pitelli, 1998; Marcondes, 2001; Velini et al., 2002; Galo et al., 2002; Cavenaghi et al., 2003; Carvalho et al., 2003).

O manejo de plantas aquáticas no país, através do controle químico (Martins et al, 1999, 2002; Neves et al., 2002; Tanaka et al., 2002; Carbonari et al., 2003; Cardoso et al., 2003; Guimarães et al., 2003; Marcondes et al., 2003; Negrisoli et al., 2003; Terra et al., 2003), controle mecânico (Velini, 1998; Antuniassi et al., 2002) e controle biológico (Miyazaki \& Pitelli, 2003; Borges Neto \& Pitelli, 2004; Borges Neto et al., 2004), são estudados e avaliados, na maioria das vezes, em sistemas fechados, como caixas-d'água e tanques fechados, ou em caráter emergencial, pois o Brasil ainda não possui legislação específica para o manejo dessas plantas em sistemas abertos (rios e reservatórios).

Dessa forma, fica evidente a importância de estudos de qualidade de água e sedimento em reservatórios e do levantamento das plantas existentes no sistema, facilitando posteriormente o manejo e o impacto que este possa causar.

A UHE Americana foi formada pela barragem do rio Atibaia. que está situado na bacia hidrográfica do rio Piracicaba. O reservatório está localizado na cidade de Americana e pertence a uma bacia hidrográfica de vasta área, com elevada atividade antrópica e grande descarga de efluentes de origem industrial e doméstica das cidades de Campinas e Paulínia, bem como intensa atividade agrícola (Leite \& Espindola, 2002).

Este trabalho teve por objetivos a caracterização de água e sedimento e o monitoramento de problemas com plantas aquáticas na UHE Americana, procurando-se correlacionar as informações obtidas.

\section{MATERIAL E MÉTODOS}

O trabalho foi realizado na UHE Americana, da Companhia Paulista de Força e Luz, localizada na cidade de Americana-SP.

Para coleta de água e sedimento foram selecionados seis pontos de amostragem no corpo do reservatório (Figura 1), sendo os pontos 1 e 6 coletados em superficie e os pontos 2, 3, 4 e 5 coletados em três profundidades (superfície, meio e fundo da seção). As campanhas de amostragens de água e sedimento foram realizadas bimestralmente, entre os meses de outubro de 2003 e junho de 2004 . Foram coletadas, no total, 70 amostras de água (14 amostras por coleta) e 30 amostras de sedimento (seis amostras por coleta) para caracterização do ambiente de ocorrência das plantas aquáticas.

Em todas as campanhas, a coleta de água foi feita com o auxílio de um coletor de fluxo contínuo, com uma mangueira de $15 \mathrm{~m}$, desenvolvido para que a amostragem fosse realizada em diferentes profundidades, succionando-se a amostra com a ajuda de uma bomba a vácuo (Figura 2a). A coleta de sedimento foi realizada com o auxílio de uma draga tipo Petersen, que era lançada em cada ponto até o fundo do reservatório (Figura $2 b$ ).

No momento da coleta foram realizadas as seguintes determinações: coordenadas geográficas do ponto, profundidade, transparência com disco de Secchi (Figura 3a), temperatura do ar e da água, teor de oxigênio dissolvido, 
$\mathrm{pH}$, condutividade elétrica, turbidez e potencial de oxirredução (Figura 3b).

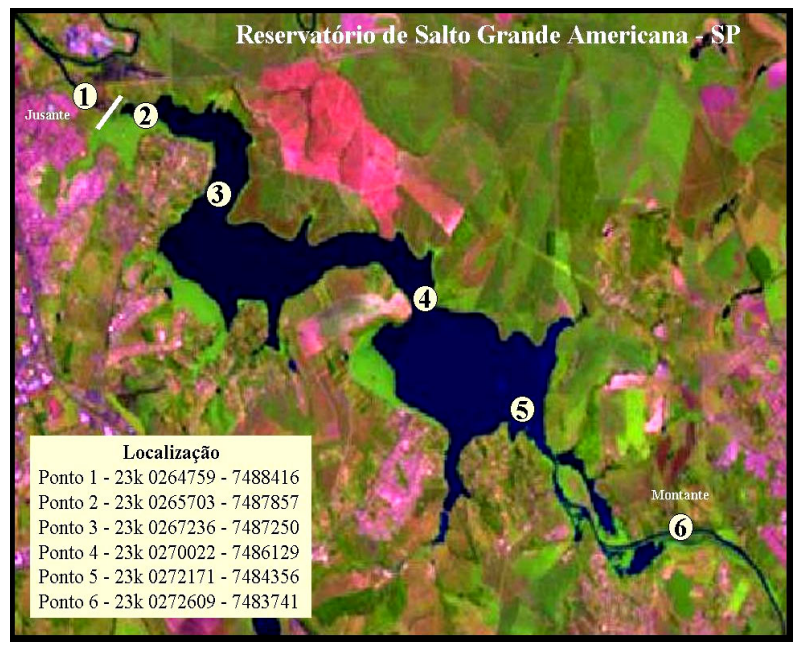

Figura 1 - Localização dos pontos na UHE Amerciana.

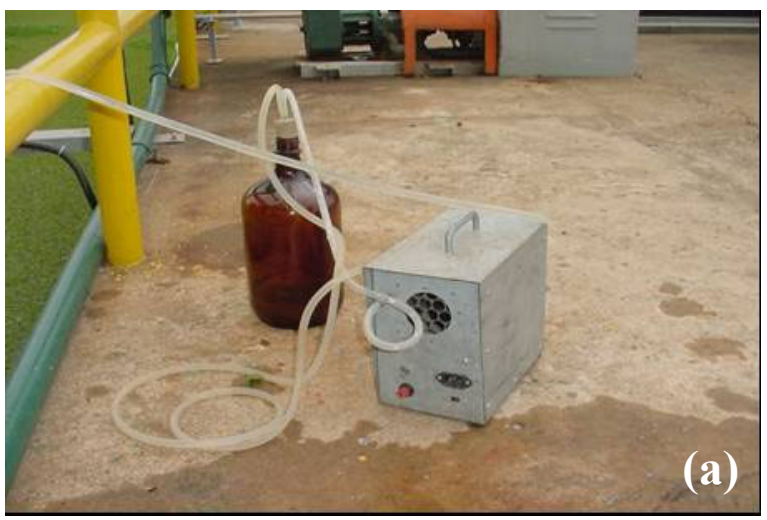

Feitas essas análises, as amostras de água e sedimento foram acondicionadas em recipientes apropriados e submetidas aos procedimentos de conservação, segundo Eaton et al. (1995).

Para cada amostra de água foram realizadas as análises de $\mathrm{pH}$; condutividade; potencial de oxirredução; turbidez; acidez; alcalinidade; teores de sólidos suspensos, dissolvidos e totais; teor de nitrato; teor de nitrito; teor de amônio; teor de nitrogênio inorgânico, orgânico e total; teor de sulfato; teores de fosfato, fósforo reativo e total; dureza; demanda química de oxigênio; demanda biológica de oxigênio; coliformes fecais e totais; fitoplâncton; microcistina; transmissão de luz para coluna d'água de $1 \mathrm{~m}$; e teores dissolvidos de $\mathrm{Na}, \mathrm{Ca}, \mathrm{Si}, \mathrm{K}, \mathrm{Cu}, \mathrm{Zn}, \mathrm{Mn}, \mathrm{Fe}, \mathrm{Ni}, \mathrm{Cd}, \mathrm{Mg}, \mathrm{Sn}$, $\mathrm{Pb}, \mathrm{Al}, \mathrm{B}, \mathrm{Ba}, \mathrm{Co}, \mathrm{Cr}, \mathrm{Hg}, \mathrm{Li}, \mathrm{Mo}$ e Se.

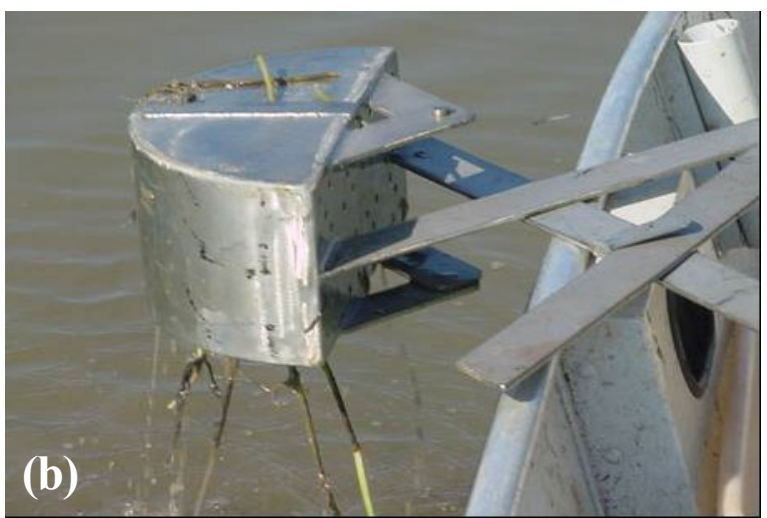

Figura 2 - Sistema de bomba a vácuo usado para coleta de amostra de água (a) e draga tipo Petersen utilizada para coleta de amostra de sedimento (b).
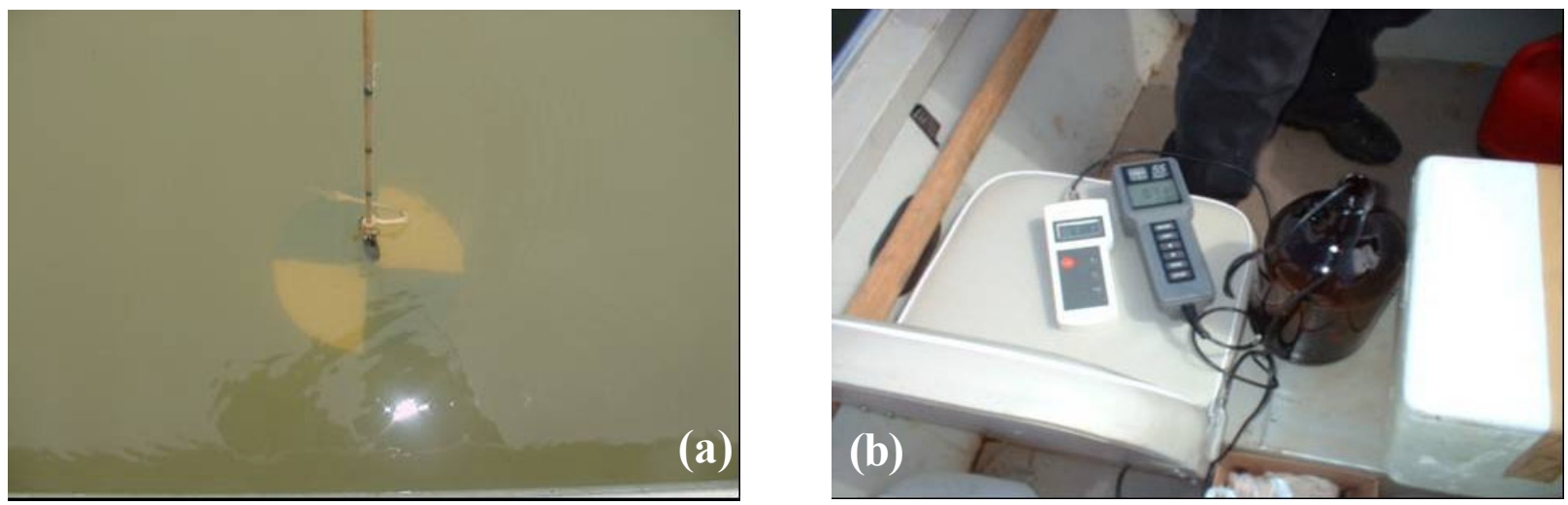

Figura 3 - Avaliação da transparência através do disco de Secchi (a) e determinação do teor de oxigênio dissolvido e pH (b). 
Em se tratando das amostras de sedimento, foram realizadas as análises de $\mathrm{pH}$, capacidade de troca de cátions (CTC), soma de bases (SB), saturação de bases (V\%), textura e teores de matéria orgânica ( $\mathrm{MO} \%), \mathrm{Ca}, \mathrm{Mg}, \mathrm{P}$, $\mathrm{K}$, nitrato, nitrito, amônia, $\mathrm{N}$ orgânico, $\mathrm{N}$ inorgânico, $\mathrm{N}$ total, $\mathrm{S}, \mathrm{H}+\mathrm{Al}$, areia, silte, argila, $\mathrm{Na}, \mathrm{Si}, \mathrm{Cu}, \mathrm{Zn}, \mathrm{Mn}, \mathrm{Fe}, \mathrm{Ni}, \mathrm{Cd}, \mathrm{Sn}, \mathrm{Pb}, \mathrm{Ag}, \mathrm{Al}$, $\mathrm{B}, \mathrm{Ba}, \mathrm{Co}, \mathrm{Cr}, \mathrm{Hg}$, Li, Mo e Se.

Os levantamentos e as identificações das plantas aquáticas foram realizados nos meses de dezembro de 2003 e abril e julho de 2004, percorrendo-se as margens do reservatório com um barco de aluminio movido por motor de popa de $25 \mathrm{HP}$. Ao longo do reservatório foram estabelecidos 20 pontos de avaliação, sendo um localizado próximo à barragem (próximo ao ponto 2 da coleta de água), outro localizado no início do reservatório (próximo ao ponto 6 da coleta de água) e os 18 pontos restantes distribuídos no reservatório. Todos os pontos foram georreferenciados através de um aparelho localizador geográfico Garmin GPS 12 e suas respectivas áreas estimadas através de um binóculo com fotômetro a laser da marca Bushnell, modelo Yardage PRO 800.

Durante o levantamento foi realizada uma estimativa visual de valor geográfico do ponto e distribuição proporcional das plantas no foco. Foram atribuídos valores de 0 a $100 \%$, tanto para as espécies presentes como para os espaços livres de macrófitas que eventualmente pudessem ocorrer dentro dos pontos amostrados. A partir desses dados foram calculados os índices de ocupação média (IOM) em todos os pontos avaliados, o que proporcionou uma estimativa da área ocupada pela espécie em todo o reservatório.

Com os dados referentes à estimativa de ocupação das espécies e dos pontos avaliados, foi determinada a freqüência relativa de cada espécie, com a utilização da fórmula proposta por Mueller-Dombois \& Ellemberg (1974): FRe $=\mathrm{FAe} / \mathrm{FAt} * 100(\%)$, em que FRe refere-se à freqüência relativa de cada espécie, FAe representa a freqüência absoluta de cada espécie $(\mathrm{FAe}=\mathrm{NAe} / \mathrm{NAt} * 100$, em que NAe significa o número de amostragens em que ocorreu uma determinada espécie e NAt é o número total de amostragens realizadas) e FAt é a somatória das freqüências absolutas de todas as espécies da comunidade infestante. Esses parâmetros possibilitaram avaliar o nível de infestação de cada espécie em toda a represa, valorizando a infestação em pontos maiores.

\section{RESULTADOS E DISCUSSÃO}

Em razão do grande número de dados gerados durante as cinco coletas realizadas (3.500 análises de água e 1.230 de sedimento), optou-se por apresentar os resultados das características mais relevantes analisadas nas amostras de água e sedimento. Nas Tabelas 1, 2 e 3 são apresentados os resultados obtidos para essas características das amostras de água. Nesse caso, optou-se também por apresentar a média dos resultados das três profundidades coletadas dos pontos 2, 3, 4 e 5 . Nas Tabelas 4 e 5 encontram-se os resultados obtidos para as análises das amostras de sedimento.

Os principais elementos responsáveis pela eutrofização de corpos hídricos são nitrogênio e fósforo. Segundo Leite \& Espíndola (2002), estudos realizados entre 1969 e 2000 demonstraram aumentos significativos nas concentrações de nitrato (de 0,326 para $0,808 \mathrm{mg} \mathrm{L}^{-1}$ ), nitrito (de 0,331 para 1,412 $\mathrm{mg} \mathrm{L}^{-1}$ ), amônia (de 0,0382 para 0,3197 $\mathrm{mg} \mathrm{L}^{-1}$ ) e fosfato total dissolvido (de 6,00 para $174,04 \mu \mathrm{g} \mathrm{L}^{-1}$ ) no reservatório da UHE Americana. Os valores médios obtidos neste trabalho para estes elementos foram de $3,867 \mathrm{mg} \mathrm{L}^{-1}$ para nitrato, $0,706 \mathrm{mg} \mathrm{L}^{-1}$ para amônia, $1,372 \mathrm{mg} \mathrm{L}^{-1}$ para nitrito e $151,979 \mu \mathrm{g} \mathrm{L}^{-1}$ para fosfato dissolvido na água. No entanto, os valores máximos observados foram 19 vezes maiores para nitrato, 15 para nitrito, 22 para amônia e 10 para fósforo total dissolvido (fosfato), em relação ao valor observado por Leite \& Espindola (2002) no ano de 2000. Os valores máximos para esses elementos foram obtidos no ponto 6, localizado na entrada do reservatório, caracterizado principalmente por transição de ambiente lótico para lêntico, grande acúmulo de plantas aquáticas e baixa profundidade devido ao avançado processo de sedimentação. Neste ponto ainda foram observados valores baixos de oxigênio dissolvido (1,56 a 3,79 $\left.\mathrm{mg} \mathrm{L}^{-1}\right)$, provavelmente em decorrência do consumo elevado deste elemento no processo de decomposição de matéria orgânica. Considerandose também os demais pontos, os valores do oxigênio dissolvido variaram entre 1,56 e 
$8,81 \mathrm{mg} \mathrm{L}^{-1}$, mas $73 \%$ dos valores ficaram abaixo de $5,0 \mathrm{mg} \mathrm{L}^{-1}$ e $50 \%$ abaixo de $4,0 \mathrm{mg} \mathrm{L}^{-1}$ - valores estes utilizados como referência para classificação de águas nas classes 2 e 3, respectivamente, da Resolução CONAMA 20 de 1986, que estabelece valores para águas destinadas ao abastecimento doméstico após tratamento convencional (BRASIL, 1986). Esses valores demonstram a intensificação de um processo de eutrofização iniciado no final da década de 1970 (Leite \& Espíndola, 2002).

O sedimento apresenta-se como um depósito de nutrientes para o sistema aquático. As maiores taxas de liberação de nutrientes do sedimento para a coluna d'água ocorrem quando a parte inferior desta encontra-se com baixas concentrações de oxigênio, ou mesmo anaeróbica. Nessas condições, o meio torna-se, do ponto de vista físico-químico, favorável à solubilização de alguns íons (Esteves, 1998). Os resultados das análises do sedimento apresentaram grande variação, devendo-se, porém, destacar o elevado nível de fertilidade dos valores médios encontrados no reservatório, sendo $32,18 \mathrm{~g} \mathrm{~kg}^{-1}$ para matéria orgânica, $68,87 \mathrm{mg} \mathrm{dm}^{-3}$ para $\mathrm{P}$, $36,96 \mathrm{mmol} \mathrm{dm}^{-3}$ para Ca e $11,88 \mathrm{mmol} \mathrm{dm}^{-3}$ para Mg. Estes nutrientes podem ser disponibilizados para coluna d'água ou mesmo retirados do sedimento diretamente pelas plantas.

Nas Tabelas 6 e 7 são apresentadas as espécies e seus valores de freqüência relativa e o índice de ocupação média de cada uma delas, respectivamente, durante as três épocas de avaliação. Espécies marginais e flutuantes foram as principais infestantes do reservatório, merecendo destaque Brachiaria subquadripara, Eichhornia crassipes, Pistia stratiotes e Salvinia auriculata. Somando as freqüências relativas dessas quatro espécies, obtêm-se os totais de 72,48, 63,71 e 70,27\%, o que significa dizer que estas quatro espécies são de ocorrência comum em todo o reservatório.

Tabela 1 - Resultados das análises de $\mathrm{pH}$, turbidez, oxigênio dissolvido, sólidos suspensos e dissolvidos para água do reservatório da UHE Americana. Coletas bimestrais realizadas entre otubro de 2003 e junho de 2004

\begin{tabular}{|c|c|c|c|c|c|c|c|}
\hline & Coleta & Ponto 1 & Ponto 2 & Ponto 3 & Ponto 4 & Ponto 5 & Ponto 6 \\
\hline \multirow{5}{*}{$\mathrm{pH}$} & Out/2003 & 7,45 & 7,60 & 8,20 & 8,40 & 7,56 & 6,22 \\
\hline & Dez/2003 & 7,11 & 7,19 & 7,27 & 7,16 & 7,11 & 7,00 \\
\hline & $\mathrm{Fev} / 2004$ & 7,26 & 7,19 & 8,19 & 8,64 & 8,51 & 7,64 \\
\hline & $\mathrm{Abr} / 2004$ & 7,17 & 7,27 & 7,09 & 6,94 & 7,12 & 6,74 \\
\hline & Jun/2004 & 6,77 & 6,80 & 6,83 & 6,85 & 6,85 & 6,88 \\
\hline \multirow{5}{*}{$\begin{array}{l}\text { Turbidez } \\
\text { (NTU) }\end{array}$} & Out $/ 2003$ & 3,10 & 9,11 & 26,62 & 53,33 & 24,48 & 21,98 \\
\hline & Dez/2003 & 21,75 & 15,38 & 20,95 & 27,33 & 53,02 & 62,00 \\
\hline & $\mathrm{Fev} / 2004$ & 3,92 & 1,57 & 24,10 & 40,63 & 26,12 & 14,84 \\
\hline & $\mathrm{Abr} / 2004$ & 1,57 & 0,63 & 0,82 & 1,13 & 2,07 & 7,07 \\
\hline & Jun/2004 & 6,58 & 5,69 & 7,19 & 9,65 & 12,87 & 11,25 \\
\hline \multirow{5}{*}{$\begin{array}{c}\text { O.D. } \\
\left(\mathrm{mg} \mathrm{L}^{-1}\right)\end{array}$} & Out $/ 2003$ & 5,44 & 2,14 & 4,49 & 6,12 & 2,36 & 1,56 \\
\hline & Dez/2003 & 3,35 & 2,58 & 6,27 & 8,81 & 6,42 & 3,07 \\
\hline & $\mathrm{Fev} / 2004$ & 4,10 & 2,76 & 5,36 & 4,77 & 4,16 & 3,13 \\
\hline & $\mathrm{Abr} / 2004$ & 4,33 & 3,26 & 5,28 & 5,00 & 4,79 & 3,55 \\
\hline & Jun/2004 & 4,54 & 3,26 & 3,28 & 3,22 & 3,94 & 3,79 \\
\hline \multirow{5}{*}{$\begin{array}{l}\text { Sólidos Suspensos } \\
\qquad\left(\mathrm{g} \mathrm{m}^{-3}\right)\end{array}$} & Out $/ 2003$ & 7,0 & 13,7 & 13,3 & 12,3 & 23,7 & 32,0 \\
\hline & Dez/2003 & 20,0 & 15,3 & 20,3 & 18,7 & 46,7 & 57,0 \\
\hline & $\mathrm{Fev} / 2004$ & 15,0 & 7,0 & 11,0 & 59,0 & 18,3 & 20,0 \\
\hline & Abr/2004" & 16,0 & 7,7 & 12,7 & 27,7 & 36,7 & 73,0 \\
\hline & Jun/2004 & 23,0 & 33,0 & 24,3 & 31,3 & 56,3 & 30,0 \\
\hline \multirow{5}{*}{$\begin{array}{l}\text { Sólidos Dissolvidos } \\
\qquad\left(\mathrm{g} \mathrm{m}^{-3}\right)\end{array}$} & Out $/ 2003$ & 303,0 & 304,0 & 308,7 & 320,7 & 461,0 & 472,0 \\
\hline & Dez/2003 & 182,0 & 165,7 & 165,0 & 157,7 & 180,0 & 181,0 \\
\hline & $\mathrm{Fev} / 2004$ & 160,0 & 158,7 & 163,3 & 170,3 & 178,3 & 186,0 \\
\hline & $\mathrm{Abr} / 2004$ & 302,0 & 298,0 & 301,0 & 293,0 & 269,3 & 389,0 \\
\hline & Jun/2004 & 256,0 & 259,7 & 255,7 & 259,3 & 292,0 & 354,0 \\
\hline
\end{tabular}


Tabela 2 - Resultados das análises de nitrato, amônio, nitrito, fosfato e fósforo total para água do reservatório da UHE Americana. Coletas bimestrais realizadas entre outubro de 2003 e junho de 2004

\begin{tabular}{|c|c|c|c|c|c|c|c|}
\hline & Coleta & Ponto 1 & Ponto 2 & Ponto 3 & Ponto 4 & Ponto 5 & Ponto 6 \\
\hline \multirow{5}{*}{$\begin{array}{l}\mathrm{NO}_{3}(\mathrm{~N}) \\
\left(\mathrm{mg} \mathrm{L}^{-1}\right)\end{array}$} & Out/2003 & 1,486 & 0,135 & 0,357 & 0,933 & 0,000 & 0,000 \\
\hline & Dez/2003 & 2,192 & 2,480 & 3,179 & 3,020 & 3,059 & 2,762 \\
\hline & $\mathrm{Fev} / 2004$ & 6,416 & 3,084 & 8,519 & 7,613 & 12,587 & 15,287 \\
\hline & $\mathrm{Abr} / 2004$ & 2,240 & 1,920 & 2,640 & 2,553 & 3,735 & 3,611 \\
\hline & Jun/2004 & 5,144 & 3,626 & 4,079 & 4,208 & 4,177 & 4,974 \\
\hline \multirow{5}{*}{$\begin{array}{l}\mathrm{NH}_{4}(\mathrm{~N}) \\
\left(\mathrm{mg} \mathrm{L}^{-1}\right)\end{array}$} & Out/2003 & 0,420 & 0,628 & 0,347 & 0,181 & 6,118 & 7,028 \\
\hline & Dez/2003 & 0,259 & 0,240 & 0,060 & 0,086 & 0,110 & 0,151 \\
\hline & $\mathrm{Fev} / 2004$ & 0,039 & 0,002 & 0,001 & 0,008 & 0,007 & 0,000 \\
\hline & $\mathrm{Abr} / 2004$ & 0,323 & 0,361 & 0,336 & 0,336 & 0,385 & 1,122 \\
\hline & Jun/2004 & 0,118 & 0,094 & 0,060 & 0,173 & 0,867 & 1,320 \\
\hline \multirow{5}{*}{$\begin{array}{l}\mathrm{NO}_{2}(\mathrm{~N}) \\
\left(\mathrm{mg} \mathrm{L}^{-1}\right)\end{array}$} & Out/2003 & 0,057 & 0,089 & 0,150 & 2,438 & 15,402 & 21,315 \\
\hline & Dez/2003 & 0,733 & 0,122 & 0,157 & 0,062 & 0,170 & 0,150 \\
\hline & $\mathrm{Fev} / 2004$ & 0,004 & 0,003 & 0,003 & 0,006 & 0,002 & 0,001 \\
\hline & $\mathrm{Abr} / 2004$ & 0,020 & 0,002 & 0,003 & 0,003 & 0,008 & 0,015 \\
\hline & Jun/2004 & 0,059 & 0,075 & 0,022 & 0,047 & 0,020 & 0,021 \\
\hline \multirow{5}{*}{$\begin{array}{l}\text { Fosfato } \\
\left(\mu \mathrm{g} \mathrm{L}^{-1}\right)\end{array}$} & Out $/ 2003$ & 16,432 & 31,801 & 4,108 & 3,603 & 1746,95 & 1624,872 \\
\hline & Dez/2003 & 20,976 & 86,105 & 55,202 & 60,89 & 10,474 & 7,383 \\
\hline & $\mathrm{Fev} / 2004$ & 13,676 & 16,829 & 14,086 & 17,216 & 22,309 & 78,532 \\
\hline & $\mathrm{Abr} / 2004$ & 8,581 & 4,983 & 5,546 & 4,831 & 31,864 & 223,802 \\
\hline & Jun/2004 & 93,616 & 20,524 & 32,175 & 31,079 & 68,842 & 202,096 \\
\hline \multirow{5}{*}{$\begin{array}{l}\text { Fósforo Total } \\
\qquad\left(\mu \mathrm{g} \mathrm{L}^{-1}\right)\end{array}$} & Out/2003 & 152,180 & 85,177 & 45,653 & 48,937 & 1946,283 & 1841,510 \\
\hline & $\mathrm{Dez} / 2003$ & 112,047 & 139,512 & 129,314 & 141,776 & 17,220 & 15,646 \\
\hline & $\mathrm{Fev} / 2004$ & 62,813 & 69,839 & 83,685 & 79,168 & 77,848 & 142,386 \\
\hline & $\mathrm{Abr} / 2004$ & 102,469 & 59,046 & 28,637 & 28,692 & 92,679 & 317,555 \\
\hline & Jun/2004 & 177,617 & 74,153 & 81,474 & 94,747 & 140,482 & 312,426 \\
\hline
\end{tabular}

Tabela 3 - Resultados das análises de sulfato, cálcio, magnésio, ferro e mercúrio para água do reservatório da UHE Americana. Coletas bimestrais realizadas entre outubro de 2003 e junho de 2004

\begin{tabular}{|c|c|c|c|c|c|c|c|}
\hline & Coleta & Ponto 1 & Ponto 2 & Ponto 3 & Ponto 4 & Ponto 5 & Ponto 6 \\
\hline \multirow{5}{*}{$\begin{array}{l}\text { Sulfato } \\
\left(\mathrm{mg} \mathrm{L}^{-1}\right)\end{array}$} & Out/2003 & 4,773 & 5,219 & 5,320 & 5,645 & 8,286 & 7,965 \\
\hline & Dez/2003 & 47,115 & 46,499 & 29,592 & 36,283 & 44,921 & 46,639 \\
\hline & $\mathrm{Fev} / 2004$ & 71,989 & 71,252 & 73,304 & 78,832 & 79,347 & 86,844 \\
\hline & $\mathrm{Abr} / 2004$ & 42,402 & 43,689 & 43,850 & 41,946 & 36,356 & 72,424 \\
\hline & Jun/2004 & 35,112 & 36,427 & 36,801 & 36,454 & 42,663 & 69,536 \\
\hline \multirow{5}{*}{$\begin{array}{c}\mathrm{Ca} \\
\left(\mathrm{mg} \mathrm{L}^{-1}\right)\end{array}$} & Out $/ 2003$ & 9,609 & 8,786 & 8,339 & 8,622 & 13,349 & 13,139 \\
\hline & Dez/2003 & 10,101 & 7,826 & 8,608 & 8,159 & 10,834 & 10,501 \\
\hline & $\mathrm{Fev} / 2004$ & 9,312 & 9,475 & 9,200 & 9,488 & 9,885 & 7,214 \\
\hline & $\mathrm{Abr} / 2004$ & 7,611 & 7,827 & 7,833 & 7,909 & 7,825 & 7,595 \\
\hline & Jun/2004 & 7,341 & 6,689 & 6,791 & 6,945 & 7,291 & 7,444 \\
\hline \multirow{5}{*}{$\begin{array}{c}\mathrm{Mg} \\
\left(\mathrm{mg} \mathrm{L}^{-1}\right)\end{array}$} & Out $/ 2003$ & 5,748 & 5,753 & 5,559 & 5,428 & 6,167 & 6,336 \\
\hline & Dez/2003 & 4,705 & 2,917 & 3,975 & 3,738 & 5,223 & 5,318 \\
\hline & $\mathrm{Fev} / 2004$ & 4,530 & 4,315 & 4,248 & 4,171 & 4,834 & 4,730 \\
\hline & $\mathrm{Abr} / 2004$ & 4,468 & 4,612 & 4,814 & 4,759 & 4,869 & 4,840 \\
\hline & Jun/2004 & 4,518 & 3,831 & 3,849 & 4,006 & 4,171 & 4,443 \\
\hline \multirow{5}{*}{$\begin{array}{c}\mathrm{Fe} \\
\left(\mathrm{mg} \mathrm{L}^{-1}\right)\end{array}$} & Out $/ 2003$ & 0,020 & 0,043 & 0,021 & 0,024 & 0,018 & 0,027 \\
\hline & Dez/2003 & 0,027 & 0,014 & 0,050 & 0,054 & 0,051 & 0,053 \\
\hline & $\mathrm{Fev} / 2004$ & 0,000 & 0,000 & 0,000 & 0,000 & 0,000 & 0,000 \\
\hline & $\mathrm{Abr} / 2004$ & 0,000 & 0,000 & 0,000 & 0,000 & 0,000 & 0,345 \\
\hline & Jun/2004 & 0,000 & 0,032 & 0,157 & 0,191 & 0,099 & 0,000 \\
\hline \multirow{5}{*}{$\underset{\left(\mathrm{mg} \mathrm{L}^{-1}\right)}{\mathrm{Hg}}$} & Out $/ 2003$ & 0,004 & 0,005 & 0,005 & 0,005 & 0,010 & 0,014 \\
\hline & Dez/2003 & 0,001 & 0,000 & 0,000 & 0,000 & 0,000 & 0,001 \\
\hline & Fev/2004 & 0,000 & 0,001 & 0,002 & 0,002 & 0,001 & 0,003 \\
\hline & $\mathrm{Abr} / 2004$ & 0,000 & 0,000 & 0,000 & 0,000 & 0,000 & 0,000 \\
\hline & Jun/2004 & 0,000 & 0,000 & 0,000 & 0,000 & 0,000 & 0,000 \\
\hline
\end{tabular}


Tabela 4 - Resultados das análises de pH, matéria orgânica, fósforo, cálcio e magnésio no sedimento do reservatório da UHE Americana. Coletas bimestrais realizadas entre outubro de 2003 e junho de 2004

\begin{tabular}{|c|c|c|c|c|c|c|c|}
\hline & Coleta & Ponto 1 & Ponto 2 & Ponto 3 & Ponto 4 & Ponto 5 & Ponto 6 \\
\hline \multirow{5}{*}{$\begin{array}{c}\mathrm{pH} \\
(\mathrm{CaCl})\end{array}$} & Out $/ 2003$ & 6,64 & 4,80 & 4,82 & 5,00 & 5,30 & 5,80 \\
\hline & $\mathrm{Dez} / 2003$ & 6,60 & 5,73 & 4,75 & 5,30 & 5,80 & 5,20 \\
\hline & $\mathrm{Fev} / 2004$ & 6,15 & 5,18 & 4,16 & 5,20 & 5,60 & 4,90 \\
\hline & $\mathrm{Abr} / 2004$ & 5,77 & 4,65 & 4,90 & 4,47 & 5,20 & 5,37 \\
\hline & Jun/2004 & 6,80 & 5,30 & 5,26 & 5,26 & 4,85 & 5,70 \\
\hline \multirow{5}{*}{$\begin{array}{l}\text { M.O. } \\
\left(\mathrm{g} \mathrm{kg}^{-1}\right)\end{array}$} & Out/2003 & 21,10 & 16,00 & 52,10 & 19,70 & 14,30 & 18,60 \\
\hline & Dez/2003 & 17,78 & 32,42 & 46,66 & 51,54 & 29,29 & 40,22 \\
\hline & $\mathrm{Fev} / 2004$ & 17,97 & 21,29 & 25,39 & 29,68 & 38,86 & 21,88 \\
\hline & $\mathrm{Abr} / 2004$ & 22,46 & 39,64 & 30,27 & 34,37 & 32,03 & 30,66 \\
\hline & Jun/2004 & 20,71 & 85,31 & 59,93 & 43,35 & 18,95 & 33,00 \\
\hline \multirow{5}{*}{$\begin{array}{c}\mathrm{P} \\
\left(\mathrm{mg} \mathrm{dm}^{-3}\right)\end{array}$} & Out/2003 & 20,40 & 10,20 & 8,50 & 6,20 & 83,40 & 27,90 \\
\hline & $\mathrm{Dez} / 2003$ & 19,70 & 36,90 & 26,90 & 34,10 & 64,00 & 52,30 \\
\hline & $\mathrm{Fev} / 2004$ & 51,67 & 6,67 & 106,56 & 58,77 & 155,22 & 85,47 \\
\hline & $\mathrm{Abr} / 2004$ & 113,59 & 74,02 & 45,49 & 142,38 & 272,42 & 133,64 \\
\hline & Jun/2004 & 31,34 & 103,19 & 124,35 & 39,12 & 30,54 & 101,00 \\
\hline \multirow{5}{*}{$\begin{array}{c}\mathrm{Ca} \\
\left(\mathrm{mmol} . \mathrm{dm}^{-3}\right)\end{array}$} & Out/2003 & 304,30 & 14,10 & 62,40 & 4,20 & 58,30 & 27,30 \\
\hline & Dez/2003 & 1,64 & 0,83 & 0,58 & 0,36 & 0,59 & 2,38 \\
\hline & $\mathrm{Fev} / 2004$ & 3,11 & 2,33 & 2,19 & 1,99 & 2,24 & 1,86 \\
\hline & $\mathrm{Abr} / 2004$ & 65,84 & 38,33 & 17,07 & 32,03 & 73,86 & 54,46 \\
\hline & Jun/2004 & 82,50 & 150,00 & 43,00 & 18,80 & 5,94 & 36,20 \\
\hline \multirow{5}{*}{$\begin{array}{c}\mathrm{Mg} \\
\left(\mathrm{mmol} \mathrm{dm}^{-3}\right)\end{array}$} & Out/2003 & 9,00 & 5,90 & 18,30 & 1,40 & 12,90 & 11,40 \\
\hline & Dez/2003 & 38,59 & 40,77 & 11,48 & 7,79 & 24,26 & 25,23 \\
\hline & $\mathrm{Fev} / 2004$ & 10,68 & 7,20 & 5,22 & 3,54 & 8,32 & 6,72 \\
\hline & $\mathrm{Abr} / 2004$ & 11,20 & 5,66 & 4,03 & 7,06 & 12,42 & 11,48 \\
\hline & Jun/2004 & 16,51 & 12,04 & 10,86 & 3,09 & 0,69 & 12,63 \\
\hline
\end{tabular}

Tabela 5 - Resultados das análises de nitrato, nitrogênio total, areia, argila e silte no sedimento do reservatório da UHE Americana. Coletas bimestrais realizadas entre outubro de 2003 e junho de 2004

\begin{tabular}{|c|c|c|c|c|c|c|c|}
\hline & Coleta & Ponto 1 & Ponto 2 & Ponto 3 & Ponto 4 & Ponto 5 & Ponto 6 \\
\hline \multirow{5}{*}{$\begin{array}{c}\mathrm{NO}_{3} \\
\left(\mathrm{mg} \mathrm{kg}^{-1}\right)\end{array}$} & Out/2003 & 36,40 & 35,00 & 28,00 & 30,80 & 30,80 & 33,60 \\
\hline & Dez/2003 & 95,20 & 134,40 & 389,20 & 295,40 & 274,40 & 462,00 \\
\hline & $\mathrm{Fev} / 2004$ & 56,00 & 58,24 & 263,76 & 64,40 & 58,24 & 195,16 \\
\hline & $\mathrm{Abr} / 2004$ & 13,83 & 14,00 & 15,23 & 14,70 & 16,98 & 14,18 \\
\hline & Jun/2004 & 76,65 & 153,13 & 41,48 & $1.38,78$ & 65,45 & 199,33 \\
\hline \multirow{5}{*}{$\begin{array}{l}\mathrm{N}_{\text {Total }} \\
\left(\mathrm{mg} \mathrm{L}^{-1}\right)\end{array}$} & Out/2003 & 630,0 & 588,0 & $3.192,0$ & 826,0 & 434,0 & 784,0 \\
\hline & Dez/2003 & 854,0 & $1.400,0$ & $2.744,0$ & $2.478,0$ & $3.206,0$ & $2.772,0$ \\
\hline & $\mathrm{Fev} / 2004$ & 826,0 & $1.764,0$ & $2.618,0$ & $1.316,0$ & $3.066,0$ & $2.394,0$ \\
\hline & $\mathrm{Abr} / 2004$ & $1.050,0$ & $2.870,0$ & $2.142,0$ & $2.534,0$ & $2.520,0$ & $2.436,0$ \\
\hline & Jun/2004 & 798,0 & $5.936,0$ & $4.424,0$ & $2.394,0$ & 560,0 & $2.226,0$ \\
\hline \multirow{5}{*}{$\begin{array}{c}\text { Areia } \\
(\%)\end{array}$} & Out $/ 2003$ & 66,5 & 61,6 & 51,7 & 79,4 & 89,1 & 64,3 \\
\hline & Dez/2003 & 33,4 & 23,1 & 28,3 & 65,3 & 1,5 & 2,1 \\
\hline & $\mathrm{Fev} / 2004$ & 49,4 & 17,0 & 0,9 & 65,3 & 9,8 & 1,3 \\
\hline & $\mathrm{Abr} / 2004$ & 57,1 & 65,5 & 73,9 & 22,7 & 23,8 & 12,8 \\
\hline & Jun/2004 & 26,0 & 16,9 & 18,7 & 71,9 & 962,0 & 12,0 \\
\hline \multirow{5}{*}{$\begin{array}{c}\text { Argila } \\
(\%)\end{array}$} & Out/2003 & 9,3 & 14,2 & 16,1 & 5,8 & 2,8 & 12,5 \\
\hline & Dez/2003 & 20,8 & 40,2 & 37,7 & 18,4 & 47,0 & 50,2 \\
\hline & $\mathrm{Fev} / 2004$ & 17,7 & 50,7 & 63,3 & 14,4 & 42,1 & 41,8 \\
\hline & $\mathrm{Abr} / 2004$ & 14,6 & 21,0 & 10,6 & 43,8 & 36,4 & 29,9 \\
\hline & Jun/2004 & 20,3 & 47,0 & 42,5 & 15,2 & 48,0 & 37,6 \\
\hline \multirow{5}{*}{$\begin{array}{l}\text { Silte } \\
(\%)\end{array}$} & Out $/ 2003$ & 24,2 & 24,2 & 32,2 & 14,8 & 8,1 & 23,2 \\
\hline & Dez/2003 & 45,8 & 36,7 & 34 & 16,3 & 51,5 & 47,7 \\
\hline & $\mathrm{Fev} / 2004$ & 32,9 & 32,3 & 35,8 & 20,3 & 48,1 & 56,9 \\
\hline & $\mathrm{Abr} / 2004$ & 33,7 & 13,5 & 15,5 & 33,5 & 39,8 & 57,3 \\
\hline & Jun/2004 & 53,7 & 63,1 & 38,8 & 12,9 & 2,6 & 50,4 \\
\hline
\end{tabular}


Tabela 6 - Valores de freqüência relativa (\%) para cada espécie durante as três épocas de avaliação

\begin{tabular}{|c|c|c|c|}
\hline \multirow{2}{*}{ Espécie } & Dez/03 & $\mathrm{Abr} / 04$ & $\mathrm{Jul} / 04$ \\
\hline & $\operatorname{FRe}(\%)$ & $\operatorname{FRe}(\%)$ & $\operatorname{FRe}(\%)$ \\
\hline Aeschynomene sensitiva & 2,75 & 4,84 & 6,25 \\
\hline Alternanthera philoxeroides & 1,83 & 1,61 & 0,00 \\
\hline Brachiaria subquadripara & 18,35 & 16,13 & 17,86 \\
\hline Commelina difusa & 0,00 & 0,81 & 0,00 \\
\hline Cyperus difformis & 2,75 & 4,03 & 0,89 \\
\hline Echinochloa polystachia & 4,59 & 2,42 & 1,79 \\
\hline Eichhornia crassipes & 17,43 & 15,32 & 16,96 \\
\hline Hedychium coronarium & 0,92 & 0,81 & 0,89 \\
\hline Ludvigia elegans & 0,00 & 0,81 & 0,00 \\
\hline Mimosa pigra & 0,92 & 0,81 & 0,89 \\
\hline Panicum rivulare & 3,67 & 3,23 & 3,57 \\
\hline Paspalum repens & 0,00 & 4,03 & 0,00 \\
\hline Pistia stratiotes & 18,35 & 16,13 & 17,86 \\
\hline Salvinia auriculata & 18,35 & 16,13 & 17,86 \\
\hline Salvinia molesta & 0,00 & 2,42 & 0,89 \\
\hline Typha angustifolia & 9,17 & 8,06 & 8,93 \\
\hline Área livre & 0,92 & 2,42 & 5,36 \\
\hline Total & 100,00 & 100,00 & 100,00 \\
\hline
\end{tabular}

Tabela 7 - Índices de ocupação médios observados para cada espécie durante as três épocas de avaliação

\begin{tabular}{|c|c|c|c|}
\hline \multirow{2}{*}{ Espécie } & Dez/03 & $\mathrm{Abr} / 04$ & $\mathrm{Jul} / 04$ \\
\hline & $\operatorname{IOM}(\%)$ & IOM (\%) & IOM $(\%)$ \\
\hline Aeschynomene sensitiva & $\overline{0,06}$ & 0,04 & $\overline{0,04}$ \\
\hline Alternanthera philoxeroides & 0,03 & 0,01 & 0,00 \\
\hline Brachiaria subquadripara & 47,76 & 67,12 & 54,29 \\
\hline Commelina difusa & 0,00 & 0,40 & 0,00 \\
\hline Cyperus difformis & 0,06 & 0,04 & 0,01 \\
\hline Echinochloa polystachia & 0,19 & 0,03 & 0,03 \\
\hline Eichhornia crassipes & 39,13 & 11,63 & 2,86 \\
\hline Hedychium coronarium & 0,03 & 0,03 & 0,03 \\
\hline Ludvigia elegans & 0,00 & 0,01 & 0,00 \\
\hline Mimosa pigra & 0,03 & 0,01 & 0,01 \\
\hline Panicum rivulare & 0,50 & 0,36 & 0,36 \\
\hline Paspalum repens & 0,00 & 0,24 & 0,00 \\
\hline Pistia stratiotes & 8,29 & 11,88 & 16,12 \\
\hline Salvinia auriculata & 1,74 & 3,87 & 8,76 \\
\hline Salvinia molesta & 0,00 & 0,09 & 0,01 \\
\hline Typha angustifolia & 1,79 & 1,84 & 2,24 \\
\hline Área livre & 0,45 & 2,45 & 15,27 \\
\hline Total & 100,00 & 100,00 & 100,00 \\
\hline
\end{tabular}

A espécie Brachiaria subquadripara foi a que mais se destacou dentro do ecossistema, pois, além de apresentar freqüência relativa alta, mostrou os maiores índices de infestação média. Juntas, Brachiaria subquadripara e Eichhornia crassipes representam $86,88 \%$ de toda a infestação de macrófitas que ocorrem no reservatório durante a primeira avaliação.
Esses resultados estão de acordo com o levantamento realizado por Faria \& Espindola (2002) no mesmo reservatório, que observaram com maior freqüência e abundância as espécies Brachiaria subquadripara, Eichhornia crassipes e Pistia stratiotes. Brachiaria subquadripara também se destacou quanto aos indices de ocupação média avaliados nas duas épocas subseqüentes, sendo estes superiores a 67 e $54 \%$ nos meses de abril e julho de 2004, respectivamente.

Embora Pistia stratiotes e Salvinia auriculata tenham demonstrado elevados valores de freqüência relativa, elas não apresentaram elevados niveis de infestação nos pontos de avaliação, mas ambas aumentaram suas áreas de ocupação no decorrer dos períodos de avaliação, com índices médios de 8,29, 11,88 e $16,12 \%$ para $P$. stratiotes e de $1,74,3,87$ e $8,76 \%$ para S. auriculata, nas avaliações de dezembro de 2003 e abril e julho de 2004, respectivamente.

As espécies encontradas são condizentes com o ambiente eutrófico apresentado pelo reservatório, que dificulta a sobrevivência de plantas submersas, em razão, principalmente, da baixa penetração de luz na coluna de água, levando à substituição dessas espécies por espécies marginais e flutuantes (Esteves, 1998; Thomaz, 2002).

\section{AGRADECIMENTOS}

À CPFL, Companhia Paulista de Força e Luz, pelo apoio técnico e financeiro. Projeto financiado com recursos da linha P \& D da ANEEL.

\section{LITERATURA CITADA}

ANTUNIASSI, U. R.; VELINI, E. D.; MARTINS, D. Remoção mecânica de plantas aquáticas: análise econômica e operacional. Planta Daninha, v. 20, p. 35-43, 2002. (Edição especial)

BORGES NETO, C. R.; PITELLI, R. A. Adjuvantes e herbicidas e a infectividade de Fusarium graminearum, agente potencial de biocontrole de Egeria densa e Egeria najas. Planta Daninha, v. 22, n. 1, p. 77-83, 2004.

BORGES NETO, C. R.; GORGATI, C. Q.; PITELLI, R. A. Influência da concentração de inóculo e da idade da planta na intensidade de doença causada por Fusarium graminearum em Egeria densa e Egeria najas. Fitopatol. Bras., v. 29, n. 3, p. 282-288, 2004. 
BRASIL. RESOLUÇÃO CONAMA No 20. Conselho Nacional de Meio Ambiente. 1986. Disponível em: <http:// www.mma.gov.br/port/conama/res/res86/res2086.html >. Acesso em: 13 dez. 2004.

CARBONARI, C. A.; MARTINS, D.; TERRA, M. A. Controle de Brachiaria subquadripara e Brachiaria mutica através de diferentes herbicidas aplicados em pós-emergência. Planta Daninha, v. 21, p. 79-84, 2003. (Edição especial)

CARDOSO, L. R.; MARTINS, D.; TERRA, M. A. Sensibilidade a herbicidas de acessos de aguapé coletados em reservatórios do estado de São Paulo. Planta Daninha, v. 21, p. 61-67, 2003. (Edição especial)

CARVALHO, F. T. et al. Plantas aquáticas e nível de infestação das espécies presentes no reservatório de Barra Bonita, no rio Tietê. Planta Daninha, v. 21, p. 15-19, 2003. (Edição especial)

CAVENAGHI, A. L. et al. Caracterização da qualidade da água e sedimento relacionados com a ocorrência de plantas aquáticas em cinco reservatórios da bacia do rio Tietê. Planta Daninha, v. 21, p. 43-52, 2003. (Edição especial)

EATON, A. D.; CLESCERI, L. S.; GREENBERG, A. E. (Eds.). Standard methods for the examination of water and wastewater. 19.ed. Maryland: American Public Health Association, 1995. $106 \mathrm{p}$.

ESTEVES, F. A. Fundamentos de limnologia. 2.ed. Rio de Janeiro: Interciência: FINEP, 1998. 575 p.

FARIA, O. B.; ESPÍNDOLA, E. L. G. Macrófitas aquáticas no Reservatório de Salto Grande (Americana, SP):

Estimativa da biomassa, concentração de nutrientes e metais. In: RECURSOS Hidroenergéticos - usos, impactos e planejamento integrado. São Carlos: 2002. p. 165-176.

GALO, M. L. B. T. et al. Uso do sensoriamento remoto orbital no monitoramento da dispersão de macrófitas nos reservatórios do complexo Tietê. Planta Daninha, v. 20, p. 7-20, 2002. (Edição especial)

GUIMARÃES, G. L. et al. Metodologia para avaliação de impacto ambiental de macrófitas em mesocosmos. Planta Daninha, v. 21, p. 37-42, 2003. (Edição especial)

LEITE, M. A.; ESPÍNDOLA, E. L. G. Análise do processo de eutrofização entre os Reservatórios de Salto Grande $\left(22^{\circ} 44^{\prime} \mathrm{S}\right.$ e $\left.47^{\circ} 15^{\prime} \mathrm{W}\right)$ e do Lobo $\left(22^{\circ} 10^{\prime} \mathrm{S}\right.$ e $\left.47^{\circ} 57^{\prime} \mathrm{W}\right)$. In: RECURSOS Hidroenergéticos - usos, impactos e planejamento integrado. São Carlos: 2002. p. 107-116.

MARCONDES, D. A. Eficiência do fluridone no controle de plantas aquáticas submersas e efeitos sobre algumas características ambientais. Botucatu, 2001. 171 f. Tese (Livre-Docência) - Universidade Estadual Paulista, Botucatu, 2001.

MARCONDES, D. A. S. et al. Eficiência de fluridone no controle de plantas aquáticas submersas no reservatório de Jupiá. Planta Daninha, v. 21, p. 69-77, 2003. (Edição especial)
MARTINS, D. et al. Controle químico de plantas daninhas aquáticas em condições controladas em caixa d'água. Planta Daninha, v. 17, n. 2, p. 289-296, 1999.

MARTINS, D. et al. Controle químico de Pistia stratiotes, Eichhornia crassipes e Salvini molesta em caixa d'água. Planta Daninha, v. 20, p. 83-88, 2002. (Edição especial)

MIYAZAKI. D. M. Y.; PITELLI, R. A. Estudo do potencial do pacu (Piaractus mesopotamicus) como agente de controle biológico de Egeria densa, Egeria najas e Ceratophyllum demersum. Planta Daninha, v. 21, p. 53-59, 2003. (Edição especial)

MUELlER-DOMBOIS, D.; ELLEMBERG, H. Aims and methods of vegetation ecology. New York: Willey \& Sons, 1974. $547 \mathrm{p}$.

NEGRISOLI, E. et al. Uso de diferentes herbicidas no controle de Muriophullum aquaticum. Planta Daninha, v. 21,p. 93-98, 2003. (Edição especial)

NEVES, T.; FOLONI, L. L.; PITELLI, R. A. Controle químico de aguapé (Eichhornia crassipes). Planta Daninha, v. 20, p. 89-97, 2002. (Edição especial)

PITELLI, R. A. Macrófitas aquáticas no Brasil, na condição de problemáticas. In: WORKSHOP SOBRE CONTROLE DE PLANTAS AQUÁTICAS, 1998, Brasília. Anais... Brasília-DF: IBAMA, 1998. p. 32-35.

TANAKA, R. H. Prejuízos provocados por plantas aquáticas. In: WORKSHOP SOBRE CONTROLE DE PLANTAS AQUÁTICAS, 1998, Brasília. Anais... IBAMA, Brasília-DF, 1998. p. 36-38.

TANAKA, R. H. et al. Avaliação de herbicidas no controle de egéria em laboratório, caixa d'água e represa sem fluxo d'água. Planta Daninha, v. 20, p. 73-81, 2002. (Edição especial)

TERRA, M. A. et al. Controle químico de plantas aquáticas: Polygonum lapathifolium. Planta Daninha, v. 21, p. 85-88, 2003. (Edição especial)

THOMAZ, S. M. Fatores ecológicos associados à colonização e ao desenvolvimento de macrófitas aquáticas e desafios de manejo. Planta Daninha, v. 20, p. 21-33, 2002. (Edição especial)

VELINI, E. D. Controle mecânico de plantas daninhas. In: WORKSHOP SOBRE CONTROLE DE PLANTAS AQUÁTICAS, 1998. Anais... Brasília-DF: IBAMA, 1998. p. 32-35.

VELINI, E. D. et al. Manejo de plantas aquáticas em grandes reservatórios: riscos associados à estratégia de não ação. In: CONGRESSO BRASILEIRO DA CIÊNCIA DAS PLANTAS DANINHAS, 23., 2002, Gramado. Resumos... Gramado: Sociedade Brasileira da Ciência das Plantas Daninhas, 2002. p. 610.

Planta Daninha, Viçosa-MG, v. 23, n. 2, p. 215-223, 2005 